\title{
A NanoBRET-Based Binding Assay for Smoothened Allows Real-time Analysis of Ligand Binding and Distinction of Two Binding Sites for BODIPY-cyclopamine ${ }^{\mathrm{S}}$
}

\author{
(D) Paweł Kozielewicz, (D)Carl-Fredrik Bowin, (D)Ainoleena Turku, and (1) Gunnar Schulte \\ Section of Receptor Biology and Signaling, Department Physiology and Pharmacology, Karolinska Institutet, Stockholm, \\ Sweden
}

Received August 28, 2019; accepted November 1, 2019

\begin{abstract}
Smoothened (SMO) is a GPCR that mediates hedgehog signaling. Hedgehog binds the transmembrane protein Patched, which in turn regulates SMO activation. Overactive SMO signaling is oncogenic and is therefore a clinically established drug target. Here we establish a nanoluciferase bioluminescence resonance energy transfer (NanoBRET)-based ligand binding assay for SMO providing a sensitive and high throughput-compatible addition to the toolbox of GPCR pharmacologists. In the NanoBRET-based binding assay, SMO is $\mathrm{N}$ terminally tagged with nanoluciferase (Nluc) and binding of BODIPY-cyclopamine is assessed by quantifying resonance energy transfer between receptor and ligand. The assay allowed kinetic analysis of ligandreceptor binding in living HEK293 cells, competition binding experiments using commercially available SMO ligands (SANT1, cyclopamine-KAAD, SAG1.3 and purmorphamine), and
\end{abstract}

pharmacological dissection of two BODIPY-cyclopamine binding sites. This high throughput-compatible assay is superior to commonly used SMO ligand binding assays in the separation of specific from non-specific ligand binding and, provides a suitable complement to chemical biology strategies for the discovery of novel SMO-targeting drugs.

\section{SIGNIFICANCE STATEMENT}

We established a NanoBRET-based binding assay for SMO with superior sensitivity compared to fluorescence-based assays. This assay allows distinction of two separate binding sites for BODIPY-cyclopamine on the SMO transmembrane core in live cells in real time. The assay is a valuable complement for drug discovery efforts and will support a better understanding of Class F GPCR pharmacology.

\section{Introduction}

Smoothened (SMO) is a G protein-coupled receptor (GPCR) that, alongside 10 paralogs of Frizzleds, forms the Class $F$ of GPCRs (Schulte, 2010). SMO signaling is of utmost importance during embryonic patterning and development, and dysfunction of SMO signaling is causative in the development of diverse tumors including basal cell carcinoma (Ingham and McMahon, 2001). Therefore, pharmacological targeting of SMO and SMO signaling evolved as an attractive antitumor treatment strategy established in clinical practice (Wu et al., 2017). On a structural level, this seven-transmembrane domain spanning receptor is characterized by a large, extracellular cysteine-rich domain (CRD) and a long C-terminal domain

The study was supported by grants from Karolinska Institutet, the Swedish Research Council (2017-04676), the Swedish Cancer Society (CAN2017/561), the Novo Nordisk Foundation (NNF17OC0026940), Stiftelsen Olle Engkvist Byggmästare (2016/193), Emil and Wera Cornells Stiftelse, and Wenner-Gren Foundations (UPD2018-0064).

The initial version of this manuscript was deposited as a preprint (https:// doi.org/10.1101/706028).

https://doi.org/10.1124/mol.119.118158.

S This article has supplemental material available at jpet.aspetjournals.org.
(Schulte, 2010). While SMO is essential for transmitting transcriptional responses via heterotrimeric $\mathrm{G}$ proteins and Glioma-associated oncogene (Gli) signaling induced by hedgehog proteins (three mammalian homologs: Desert, Indian, and Sonic hedgehog), the nature and mode of action of the endogenous ligand and the mechanisms of receptor activation are not fully understood (Byrne et al., 2016; Kong et al., 2019; Schulte and Kozielewicz, 2019). It is known that hedgehog proteins bind Patched, a cholesterol transporter, which in turn regulates SMO activation by postulated regulation of cholesterol levels (Zhang et al., 2018b). Cholesterol and other naturally occurring sterols are positive allosteric modulators and agonists of SMO in Gli and G protein-dependent signaling (Nachtergaele et al., 2012; Sever et al., 2016; Raleigh et al., 2018; Kowatsch et al., 2019; Qi et al., 2019). Moreover, recently solved structures of SMO in its active conformation have provided valuable insight into ligand-induced activation mechanism of the Class F receptor (Deshpande et al., 2019; Qi et al., 2019).

Due to the distinct link to human cancer and occurrence of several cancer-associated SMO mutations [e.g., $\mathrm{D} 473 \mathrm{H}^{6.54}$ or W535L ${ }^{7.55}$; superscript numbering refers to Ballesteros Weinstein nomenclature of GPCRs (Ballesteros and Weinstein,

ABBREVIATIONS: BSA, bovine serum albumin; $\mathrm{Cl}$, confidence interval; CRD, cysteine-rich-domain; PDL, poly-D-lysine; ELISA, enzyme-linked immunosorbent assay; Gli, glioma-associated oncogene; GPCR, G protein-coupled receptor; Hh, hedgehog; NanoBRET, nanoluciferase-based bioluminescence resonance energy transfer; Nluc, nanoluciferase; PBS, phosphate-buffered saline; SMO, Smoothened; 7TM, seven transmembrane. 
1995)], a plethora of small ligands, antagonists and inverse agonists, has been developed to target this receptor (Wu et al., 2017). Three of these compounds, vismodegib, sonidegib, and glasdegib, are approved as drugs for the treatment of basalcell carcinoma (vismodegib and sonidegib) and acute myeloid leukemia (glasdegib) (Chen, 2016; Hoy, 2019). In addition, nature provides an effective SMO antagonist, the plant alkaloid cyclopamine (Incardona et al., 1998; Taipale et al., 2000). These and other ligands, such as SMO agonists (e.g., SAG series of analogs, purmorphamine), neutral antagonists (e.g., SANT-1), or inverse agonists (e.g., cyclopamine-KAAD), are frequently used to explore SMO pharmacology (Chen et al., 2002b; Rominger et al., 2009; Chen, 2016). There are two binding pockets on the transmembrane core of SMO (Wang et al., 2014). The upper binding pocket can accommodate ligands, such as SAG or vismodegib, whereas the lower binding pocket binds, e.g., SANT-1. To date, binding affinities of the SMO ligands were determined using classic radioligand binding methods (Frank-Kamenetsky et al., 2002; Rominger et al., 2009; Wang et al., 2014) and, more often, fluorescencebased assays using the green-yellow fluorescent BODIPYcyclopamine (Chen et al., 2002a,b; Manetti et al., 2010; Gorojankina et al., 2013; Huang et al., 2016, 2018). The fluorescently labeled cyclopamine has been used in three separate experimental approaches: assessment of ligandreceptor interaction based on detection of fluorescence using confocal microscopy, flow cytometry, or fluorescence polarization (Chen et al., 2002a,b; Bee et al., 2012; Gorojankina et al., 2013; Huang et al., 2016, 2018; Lu et al., 2018). While these methods offer valuable insight into ligand-receptor binding in live cells, they suffer from several shortcomings, including 1) laborious protocols that require long ligand incubation times (up to 10 hours); 2) extensive cell washing due to lipophilicity of BODIPY-cyclopamine; 3) high levels of non-specific binding in untransfected cells or in the presence of saturating concentrations of unlabeled competitors; 4) the need for data normalization of BODIPY-fluorescence values to receptor expression values; and 5) in the case of radioligand binding experiments, health risks, need for well-controlled designated areas, and waste disposal.

To overcome these experimental limitations, we established and validated a live-cell, nanoluciferase bioluminescence resonance energy transfer (NanoBRET)-based binding assay to assess the binding properties of BODIPY-cyclopamine and unlabeled SMO ligands to an $\mathrm{N}$ terminally nanoluciferase (Nluc)-tagged SMO (Nluc-SMO) and SMO lacking the CRD ( $\triangle$ CRD Nluc-SMO) in HEK293 cells devoid of endogenous SMO ( $\Delta$ SMO HEK293). This proximity-based ligand-binding assay was developed recently to assess ligand binding to Class A GPCRs and receptor tyrosine kinases (Stoddart et al., 2015, 2018a,b; Mocking et al., 2018; Bosma et al., 2019; BouzoLorenzo et al., 2019; Sykes et al., 2019). It relies on the high specificity of BRET between Nluc-tagged protein (BRET donor) and fluorescently tagged ligand (BRET acceptor) that can only occur when both BRET partners are within a distance of $10 \mathrm{~nm}$ (100 ̊). Thus, the interference of non-specifically bound probe, outside of the BRET radius to the receptor is, in contrast to detecting solely ligand fluorescence, minimal. Along these lines, no washing steps are required. In the present study, we employ NanoBRET to monitor binding of commercially available SMO ligands. Furthermore, the sensitivity of the assay enabled us to dissect the pharmacological properties of separate BODIPY- cyclopamine binding pockets in the transmembrane-spanning receptor core of the Class $\mathrm{F}$ receptor SMO. Thus, this NanoBRET-based binding assay provides a valuable complement to the toolbox of high-throughput compatible screening assays for Class F GPCRs.

\section{Materials and Methods}

DNA Cloning and Mutagenesis. Nluc- $\mathrm{A}_{3}$ was from Stephen Hill, University of Nottingham, UK (Stoddart et al., 2015). SMO-Rluc8 coding for mouse Smoothened was from Nevin A. Lambert, Augusta University, Georgia (Wright et al., 2019). The mouse SMO sequence was subcloned into an empty $\mathrm{N}$ terminally tagged Nluc vector containing $5-\mathrm{HT}_{3} \mathrm{~A}$ signal peptide using BamHI and $\mathrm{XbaI}$ restriction sites. First, the BamHI site present in mouse SMO was removed using site-directed mutagenesis (GeneArt; Thermo Fisher Scientific) with the following primers: 5 -CCTCCAGGGGCTGGGGTCCATTCATTC CCGC-3' (forward primer) and 5'-GCGGGAATGAATGGACCCCAG CCCCTGGAGG-3' (reverse primer). Next, the mouse SMO sequence was cloned in-frame into the Nluc vector using forward primer: $5^{\prime}$-GA CGGATCCGCGGCCTTGAGCGGGAACGTG-3' and reverse primer: 5'-CGTTCTAGATCAGAAGTCCGAGTCTGCATC-3'. $\Delta$ CRD NlucSMO was generated using the mouse SMO lacking the BamHI site by cloning it into $\mathrm{N}$ terminally tagged Nluc vector between BamHI and XbaI using forward primer: 5'-GACGGATCCGAGGTACAAAACATC AAGTTC-3; and reverse primer: 5'-CGTTCTAGATCAGAAGTCCGA GTCTGCATC-3'. $\triangle$ CRD and full-length Nluc-SMO D477G ${ }^{6.54} /$ E522K ${ }^{7.38}$ were generated with site-directed mutagenesis (GeneArt; Thermo Fisher Scientific) by first obtaining D477G ${ }^{6.54}$ mutation with the following primers: 5'-GCTGCCACTTCTATGGCTTCTTCAACC AGGC-3' (forward primer) and 5'-GCCTGGTTGAAGAAGCCATAG AAGTGGCAGC-3' (reverse primer). Subsequently the E522K ${ }^{7.38}$ mutation was introduced with $5^{\prime}$-CCCAGCCTCCTGGTGAAGAAG ATCAATCTAT-3' (forward primer) and 5'-ATAGATTGATCTTCT TCACCAGGAGGCTGGG-3 (reverse primer). All constructs were validated by sequencing (Eurofins GATC, Konstanz, Germany).

Cell Culture. $\triangle$ SMO HEK293 cells were generated with CRISPR/ Cas9 genome editing using SMO-targeting sgRNA 5'-CAACCCCAA GAGCTGGTACGAGG-3'. The cells were cultured in DMEM (HyClone) supplemented with $10 \%$ fetal bovine serum, $1 \%$ penicillin/ streptomycin, and 1\% L-glutamine (all Thermo Fisher Scientific) in a humidified $\mathrm{CO}_{2}$ incubator at $37^{\circ} \mathrm{C}$. To generate cell lines stably expressing Nluc-SMO and $\Delta$ CRD Nluc-SMO, $\Delta$ SMO HEK293 cells were transfected with Nluc-SMO and $\Delta$ CRD Nluc-SMO constructs using Lipofectamine 2000 (Thermo Fisher Scientific), according to the manufacturer's instructions. About 24 hours post transfection, cells were passaged at 1:10, and 48 hours post transfection medium was supplemented with $2000 \mu \mathrm{g} / \mathrm{ml}$ geneticin (Thermo Fisher Scientific). The medium was replaced every 2 days to select the cells transfected with the plasmids. The cells were maintained in the presence of the antibiotic for a period of 3 weeks until the stable culture was established. Absence of mycoplasma contamination was routinely confirmed by PCR using $5^{\prime}$ GGCGAATGGGTG AGTAACACG-3' and 5'-CGGATAACGCTTGCG ACTATG-3' primers detecting $16 S$ ribosomal RNA of mycoplasma in the media after 2 to 3 days of cell exposure. All cell culture plastics were from Sarstedt, unless otherwise specified.

Live-Cell ELISA. For quantification of cell surface receptor expression by labeling with anti-Nluc antibody, $\triangle$ SMO HEK293 cells at the density of $4 \cdot \times 10^{5}$ cells $/ \mathrm{ml}$ were transfected in suspension using Lipofectamine 2000 with $50-500 \mathrm{ng}$ of the indicated receptor plasmid DNA with 500-950 ng of pcDNA plasmid DNA. The cells $(100 \mu \mathrm{l})$ were seeded onto a PDL (poly-D-lysine)-coated transparent 96-well plate with flat bottom and grown overnight. Twenty-four hours later, the cells were washed twice with $0.5 \%$ BSA in PBS and incubated with a mouse anti-Nluc $(2 \mu \mathrm{g} / \mathrm{ml}$, \#MAB10026; RnD Systems) in 1\% BSA/ PBS for 1 hour at $4^{\circ} \mathrm{C}$. Following incubation, the cells were washed four times with $0.5 \% \mathrm{BSA} / \mathrm{PBS}$ and incubated with a horseradish 
peroxidase-conjugated goat anti-mouse antibody (1:3000, \#31430; Thermo Fisher Scientific) in $1 \% \mathrm{BSA} / \mathrm{PBS}$ for 1 hour at $4^{\circ} \mathrm{C}$. The cells were washed three times with $0.5 \% \mathrm{BSA} / \mathrm{PBS}$, and $50 \mu \mathrm{l}$ of the peroxidase substrate 3,3',5,5'-tetramethylbenzidine (\#T8665; SigmaAldrich, St. Louis, MO, USA) were added. The cells were incubated further for 20 minutes, and upon development of a blue product, $50 \mu \mathrm{l}$ of $2 \mathrm{M} \mathrm{HCl}$ was added and the absorbance was read at $450 \mathrm{~nm}$ using a BMG $\Omega$ POLARstar plate reader. The data were analyzed in GraphPad Prism 6.

Immunoblotting. $\triangle$ SMO HEK293 cells were transfected in suspension using Lipofectamine 2000 (50-500 ng of receptor plasmid DNA with 500-950 ng of pcDNA plasmid DNA per $4 \cdot \times 10^{5} \mathrm{cells} / \mathrm{ml}$ ) and seeded $(700 \mu \mathrm{l})$ onto wells of a 24 -well plate. Protein lysates were obtained using Laemmli buffer with $0.5 \%$ NP-40 and $5 \% \beta$-mercaptoethanol. Lysates were sonicated and analyzed on $4 \%-20 \%$ Mini-PROTEAN TGX precast polyacrylamide gels (BioRad) and transferred to PVDF membranes using the Trans-Blot Turbo system (Bio-Rad). After blocking with 5\% milk in TBS-T, membranes were incubated with primary antibodies in blocking buffer: rabbit anti-GAPDH (1:5000, \#2118; Cell Signaling Technology) and mouse anti-Nluc (0.5 $\mu \mathrm{g} / \mathrm{ml}$, \#MAB10026; RnD Systems) overnight at $4^{\circ} \mathrm{C}$. Proteins were detected with horseradish peroxidaseconjugated secondary antibody [1:5000; goat anti-rabbit (\#31460; Thermo Fisher Scientific)or 1:3000; goat anti-mouse (\#31430; Thermo Fisher Scientific), and Clarity Western ECL Blotting Substrate (Bio-Rad)]. All uncropped blots can be found in the Supplemental Fig. 1.

NanoBRET Binding Assay. $\triangle$ SMO HEK293 cells were transiently transfected in suspension using Lipofectamine 2000 (Thermo Fisher Scientific). Cells $\left(4 \times 10^{5}\right.$ cells $\left./ \mathrm{ml}\right)$ were transfected with $50-500 \mathrm{ng}$ of receptor plasmid DNA and 500-950 ng of pcDNA. The cells $(100 \mu \mathrm{l})$ were seeded onto a PDL-coated black 96 -well cell culture plate with solid flat bottom (Greiner Bio-One). Twenty-four hours post-transfection, cells were washed once with HBSS (HyClone) and maintained in the same buffer. In the saturation experiments, the cells were incubated with different concentrations of BODIPYcyclopamine $(80 \mu \mathrm{l})$ for 60 minutes at $37^{\circ} \mathrm{C}$ before the addition of the luciferase substrate coelenterazine $\mathrm{h}(5 \mu \mathrm{M}$ final concentration, $10 \mu \mathrm{l})$ for 6 minutes prior to the BRET measurement. In the competition experiments, the cells were preincubated with different concentrations of unlabeled ligands $(70 \mu \mathrm{l})$ for 30 minutes at $37^{\circ} \mathrm{C}$. Fixed concentration of BODIPY-cyclopamine was then added (10 $\mu \mathrm{l})$ and the cells were incubated for an additional 60 minutes at $37^{\circ} \mathrm{C}$ before the addition of the luciferase substrate coelenterazine-h ( $5 \mu \mathrm{M}$ final concentration, $10 \mu \mathrm{l}$ ) for 6 minutes prior to the BRET measurement. In the association experiments, the cells were preincubated with $10 \mu \mathrm{M}$ SANT-1 (30 minutes), followed by coelenterazine-h $\mathrm{h}(5 \mu \mathrm{M}$ final concentration) at $37^{\circ} \mathrm{C}$ prior to the addition of different BODIPY-cyclopamine concentrations. The BRET signal was measured every minute for 90 minutes at $37^{\circ} \mathrm{C}$. The BRET ratio was determined as the ratio of light emitted by BODIPYcyclopamine (energy acceptor) and light emitted by Nluc-tagged biosensors (energy donors). The BRET acceptor (bandpass filter 535-30 nm) and BRET donor (bandpass filter 475-30 nm) emission signals were measured using a CLARIOstar microplate reader (BMG). $\triangle$ BRET ratio was calculated as the difference in BRET ratio of cells treated with ligands and cells treated with vehicle. BODIPY fluorescence was measured prior to reading luminescence (excitation: $477-14 \mathrm{~nm}$, emission: $525-30 \mathrm{~nm}$ ). To calculate Z-factors (Zhang et al., 1999), $\triangle$ SMO HEK293 stably overexpressing Nluc-SMO or $\triangle$ CRD Nluc-SMO were plated onto PDL-coated 96-well plates. On the next day, the cells were pre-incubated either with vehicle ( $0.1 \%$ dimethylsulfoxide, 48 wells) or $10 \mu \mathrm{M}$ SANT-1 (48 wells) for 30 minutes at $37^{\circ} \mathrm{C}$ prior to the addition of BODIPY-cyclopamine $(200 \mathrm{nM}$ for Nluc-SMO stable cells and $10 \mathrm{nM}$ for $\triangle \mathrm{CRD}$ Nluc-SMO stable cells). The following equation was used to calculate Z-factor:
$Z-$ factor $=1-\frac{3\left(S D\left(\text { raw BRET } \text { ratio }_{\text {vehicle }}\right)+S D\left(\text { raw BRET ratio } 10_{\mu \mathrm{M} \mathrm{SANT}-1}\right)\right.}{\mid \text { mean }\left(\text { raw BRET } \text { ratio }_{\text {vehicle }}\right)-\text { mean }\left(\text { raw BRET ratio }_{10} \mu \mathrm{M} \mathrm{SANT}-1\right)}$

Data were analyzed using GraphPad Prism 6.

Computational Studies. Molecular docking was conducted with Glide SP (2018-4; Schrödinger LLC, New York, 2018) using default parameters. BODIPY-cyclopamine was docked to a $25 \times 25 \times 25 \AA^{3}$ box located either on the mass center of SAG21k (i.e., the upper binding pocket) or to the mass center of both SAG21k and the 7TMbound cholesterol (i.e., the lower binding pocket) of SMO [PDB ID: 6O3C (Deshpande et al., 2019)]. Prior to docking, the SMO structure was prepared with protein preparation wizard of Schrödinger Maestro and BODIPY-cyclopamine conformations generated using LigPrep with Epik in $\mathrm{pH} 7 \pm 2$ (Shelley et al., 2007). The protocol was tested by docking cyclopamine to the same SMO structure (PDB ID: 6O3C) and comparing it to the cyclopamine-SMO complex (PDB ID: 4O9R, Supplemental Fig. 2). It reproduced a similar cyclopamine binding pose to that in the crystal structure (Supplemental Fig. 2). The active SMO has a larger binding site compared with the inactive SMO; this may contribute to the ligand $\mathrm{RMSD}=2.55 \AA$ (the highest scoring pose, Glide DockingScore).

Solvent-accessible surface areas were calculated and solventaccessible surfaces visualized with Biovia DiscoverStudio Visualizer 2017 R2 (Dassault Systèmes SE) using 960 grid points per atom and probe radius of $1.40 \AA$. Active SMO structure (PDB ID: 6O3C) was used as a representative of a full-length SMO, whereas active $\triangle$ CRD-SMO structure [PDB ID: 6OT0 (Qi et al., 2019)] was used as a representative of a $\triangle \mathrm{CRD}-\mathrm{SMO}$. $6 \mathrm{O} 3 \mathrm{C}$ and $6 \mathrm{OT} 0$ were selected for the calculation as they represent the same conformational state (ligand-bound, active) of the receptor and offer thus the best comparability between the currently solved SMO and $\triangle$ CRD SMO structures.

Ligands. BODIPY-cyclopamine was from BioVision Inc. Purmorphamine (9-cyclohexyl- $N$-[4-(4-morpholinyl)phenyl]-2-(1-naphthalenyloxy)$9 \mathrm{H}$-purin-6-amine) was from Abcam. SAG1.3 (3-chloro- $N$-[trans-4(methylamino)cyclohexyl]- $N$-[[3-(4-pyridinyl)phenyl]methyl]benzo [b] thiophene-2-carboxamide) was from Sigma. Cyclopamine-KAAD and SANT-1 ((4-benzyl-piperazin-1-yl)-(3,5-dimethyl-1-phenyl-1Hpyrazol-4-ylmethylene)-amine) were from Abcam. All ligands were dissolved in dimethylsulfoxide and stored in aliquots at $-20^{\circ} \mathrm{C}$. The ligands underwent a maximum of two freeze-thaw cycles. Coelenterazine-h was from Biosynth and it was stored as $2.4 \mathrm{mM}$ aliquots in acidified ethanol at $-80^{\circ} \mathrm{C}$. Protein-low binding tubes (Eppendorf) were used to make serial dilutions of BODIPY-cyclopamine.

Data and Statistical Analysis. Live-cell ELISA data were analyzed using GraphPad Prism 6 and represent mean \pm S.E.M. of $n$ individual experiments (biological replicates) performed at least in duplicates (technical replicates). Live-cell ELISA data were analyzed for differences with one-way ANOVA with Fisher's least significant difference post hoc analysis. Significance levels are given as $* P<0.05$; ** $P<0.01$; $* * * P<0.001$; $* * * * P<0.0001$. Please refer to the figure legends for more details on the displayed data.

BODIPY-cyclopamine saturation curves were fit using threeparameter or biphasic nonlinear regression models (logarithmic scale for BODIPY-cyclopamine concentrations) or one- or two-site saturation nonlinear regression models (linear scale for BODIPYcyclopamine concentrations). Error bars on the binding curves represent mean \pm S.E.M. from $n$ independent experiments for each tested concentration. Affinity values obtained from logarithmic scale data are presented as a best-fit $\mathrm{p} K_{\mathrm{d}}\left(\mathrm{p} K_{\mathrm{i}}\right.$ for unlabeled ligands $) \pm$ S.D. Maximal binding values obtained from linear data are presented as a best-fit $B_{\max }$ with $95 \%$ confidence interval (CI). NanoBRET binding models were selected based on an extra-sum-of square $F$-test $(P<0.05)$.

Competition binding curves were analyzed using a one-site competitive binding model to obtain equilibrium dissociation constants 
values $\left(K_{\mathrm{i}}\right)$ of unlabeled ligands as per the modified Cheng-Prusoff equation (Cheng and Prusuff, 1973):

$$
\log \mathrm{IC}_{50}=\log \left(10^{\log K_{\mathrm{i}} *\left(1+\frac{(\mathrm{LL})}{K_{\mathrm{d}}}\right)}\right)
$$

where $K_{\mathrm{i}}$ is the searched dissociation constant of an unlabeled ligand, $\mathrm{IC}_{50}$ is the inhibitory constant 50 of an unlabeled ligand obtained from the competition curve, [LL] is the concentration of a labeled ligand used in the competition experiment, and $K_{\mathrm{d}}$ is the equilibrium dissociation constant of a labeled ligand obtained from the saturation studies.

To analyze the labeled ligand binding kinetics data, one-phase association or two-phase association models were selected based on an extra-sum-of square $F$-test:

One-phase association:

$$
Y=Y_{0}+\left(\text { plateau }-Y_{0}\right) *\left(1-e^{\left(-k_{\mathrm{obs}} * x\right)}\right)
$$

Two-phase association:

$Y=Y_{0}+\operatorname{Span}$ fast $*\left(1-e^{\left(-k_{\mathrm{obs}} \text { fast } * x\right)}\right)+\operatorname{Span} \operatorname{slow} *\left(1-e^{\left(-k_{\mathrm{obs}} \text { slow } * x\right)}\right)$

where $Y_{0}$ is $Y$ value at time $x=0$, plateau is the $Y$ value at infinite times, and $k_{\text {obs }}$ is the association constant expressed in $\frac{1}{\min }, t_{1 / 2}=\ln (2) /$ $k_{\text {obs }}$.

$k_{\text {on }}$ (Association rate) and $k_{\text {off }}$ (dissociation rate) are calculated from the following linear equation:

$$
k_{\mathrm{obs}}=k_{\mathrm{off}}+k_{\mathrm{on}} *[\mathrm{LL}]
$$

"Kinetic" $K_{\mathrm{d}}$ is calculated using $k_{\mathrm{on}}$ and $k_{\text {off }}$ and represented with \pm S.D.:

$$
K_{\mathrm{d}}=\frac{k_{\mathrm{of} f}}{k_{\mathrm{on}}}
$$

\section{Results}

N Terminally Nluc Tagged SMO Constructs Are Expressed at the Cell Surface. To establish a NanoBRET-based binding assay for the Class F receptor SMO, we adopted the cloning strategy of previously presented Class A GPCR including a $5-\mathrm{HT}_{3} \mathrm{~A}$ receptor-derived signal sequence and an extracellular, $\mathrm{N}$ terminally Nluc fused to either the full-length mouse SMO or $\triangle$ CRD SMO. Subsequently, these constructs are referred to as Nluc-SMO and $\triangle$ CRD Nluc-SMO, respectively (Fig. 1A). Both receptor constructs are expressed in the cells and at the cell surface upon transient transfection of $\triangle$ SMO HEK293 cells as shown by immunoblotting of whole cell lysates and a live-cell surface ELISA (Fig. 1, B and C).

BODIPY-cyclopamine Binding to Nluc-SMO Can Be Monitored by NanoBRET. The commercially available BODIPY-labeled derivative of the plant alkaloid cyclopamine (BODIPY-cyclopamine; Fig. 2A) associates with Nluc-SMO transiently expressed in $\triangle$ SMO HEK293 cells in a concentration-dependent manner, reaching saturation at $\sim 1000 \mathrm{nM}$ $\left(\mathrm{p} K_{\mathrm{d}}=6.8 \pm 0.1\right.$, Fig. $2, \mathrm{~B}$ and $\left.\mathrm{C}\right)$, which is consistent with recently published data (Lu et al., 2018). Similarly, BODIPY-cyclopamine binds the $\triangle \mathrm{CRD}$ SMO construct (biphasic fit $\mathrm{p} K_{\mathrm{d} 1}=8.4 \pm 0.2$, Fig. $2, \mathrm{D}$ and $\mathrm{E}$ ) with higher affinity and importantly the NanoBRET produced by BODIPY-cyclopamine binding was larger in the $\triangle$ CRD Nluc-SMO compared with

\section{A}

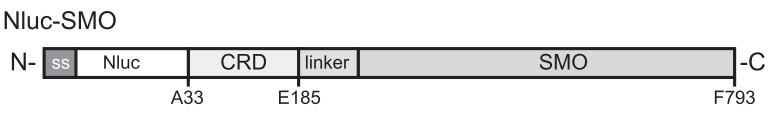

$\triangle$ CRD NluC-SMO
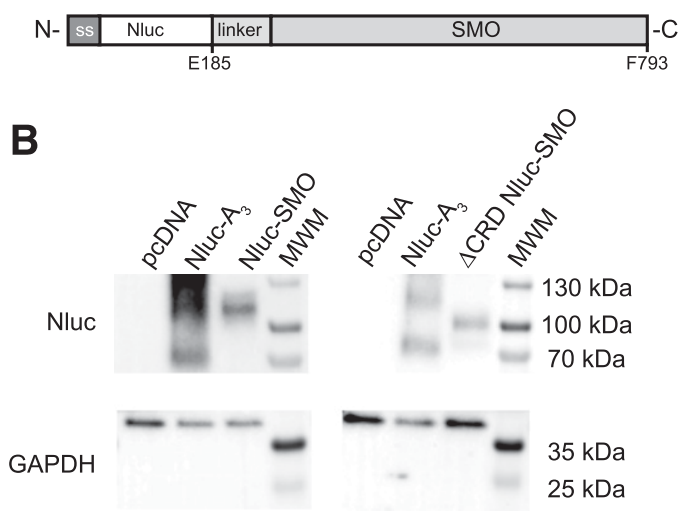

C
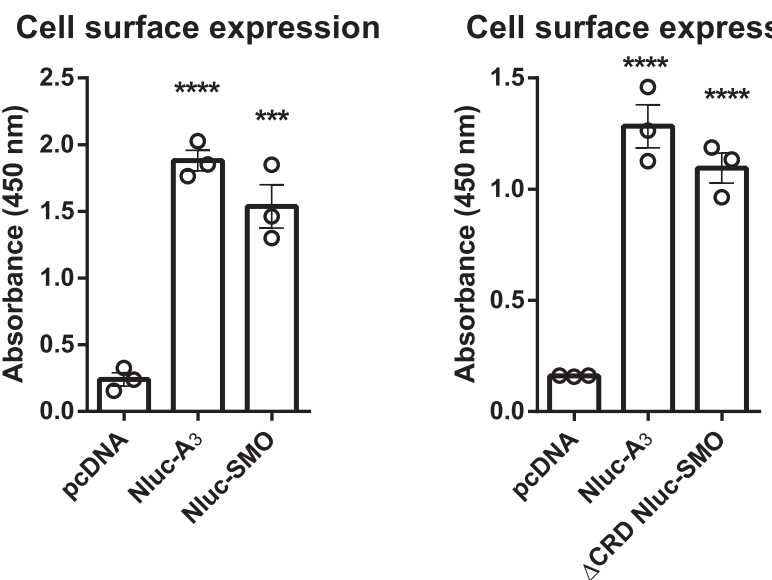

Fig. 1. Construct validation of the $\mathrm{N}$ terminally tagged Nluc-SMO. (A) Schematic presentation of the NanoBRET Nluc-SMO and $\triangle$ CRD NlucSMO sensors for BODIPY-cyclopamine binding. (B) Validation of cellular expression of Nluc-SMO and $\triangle$ CRD Nluc-SMO upon transient transfection into $\triangle$ SMO HEK293 cells. Nluc- $\mathrm{A}_{3}$ was used as a positive control. Cell lysates were analyzed by immunoblotting using anti-Nluc antibody and anti-GAPDH served as a loading control. The higher apparent molecular weights of Nluc- $\mathrm{A}_{3}$ (predicted molecular weight $=55 \mathrm{kDa}$ ), Nluc-SMO (predicted molecular weight $=103 \mathrm{kDa}$ ) and $\triangle \mathrm{CRD}$ Nluc-SMO (predicted molecular weight $=87 \mathrm{kDa}$ ) could be a result of $N$-glycosylation of the receptors. The experiments were repeated three times with similar results. (C) Surface expression of Nluc-SMO (left) and $\triangle$ CRD Nluc-SMO (right) was quantified by ELISA based on labeling with an anti-Nluc antibody. Raw data are shown from $n=3$ individual experiments and are presented as mean \pm S.E.M.; $* * * P<0.001, * * * * P<0.0001$ (one-way ANOVA).

Nluc-SMO (two-sites fit $\triangle \mathrm{BRET} \mathrm{B}_{\max 1} \triangle \mathrm{CRD}$ receptor $=$ $0.080,95 \%$ CI [0.07-0.09] and one-site fit $\triangle$ BRET $B_{\max }$ fulllength receptor $=0.034,95 \%$ CI $[0.031-0.036]$ ). Furthermore, BODIPY-cyclopamine binding to $\triangle \mathrm{CRD}$ Nluc-SMO was more complex than binding to Nluc-SMO as indicated by the biphasic binding curve for $\triangle$ CRD Nluc-SMO especially at higher concentrations of the ligand (biphasic fit $\mathrm{p} K_{\mathrm{d} 2}=6.8 \pm 0.7$ and $\triangle$ BRET $B_{\max 2}=0.033,95 \%$ CI $[0.021-0.045]$ ).

While quantification of BODIPY-cyclopamine binding on living cells was assessed 60 minutes after ligand addition, we were also interested in the binding kinetics of BODIPYcyclopamine. Therefore, we followed BODIPY-cyclopamine 
A

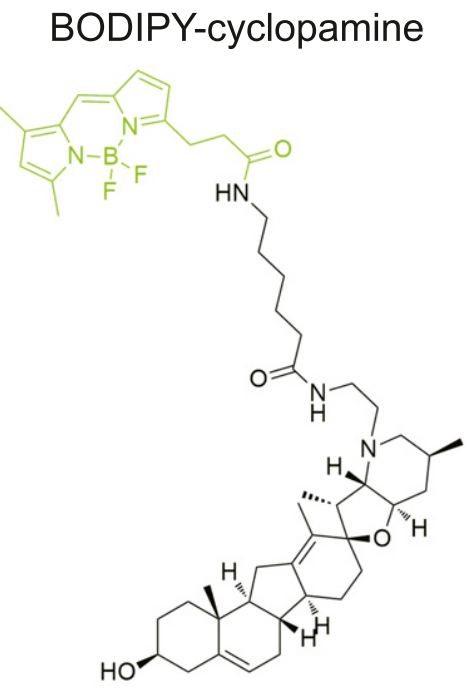

B

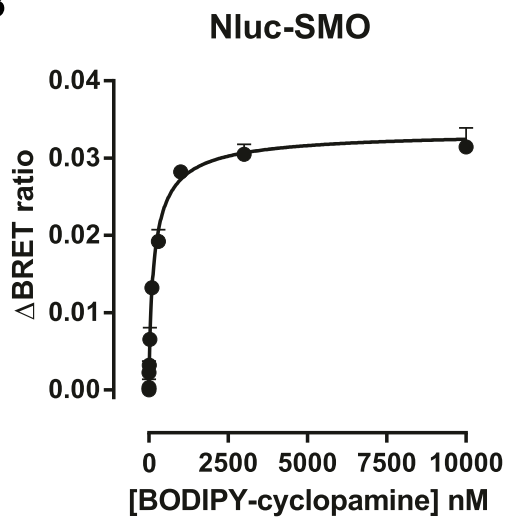

D

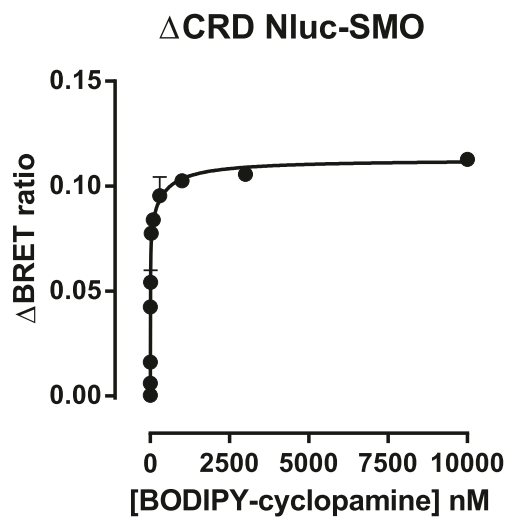

C

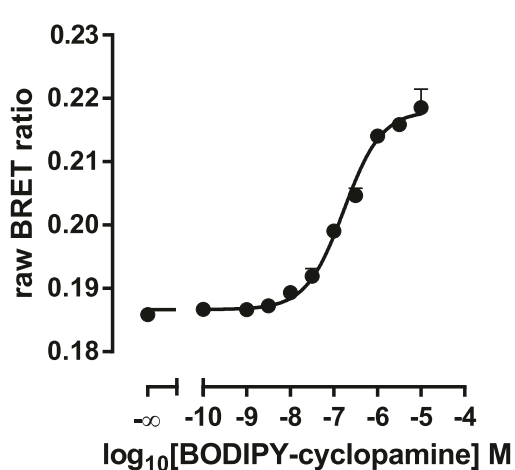

E

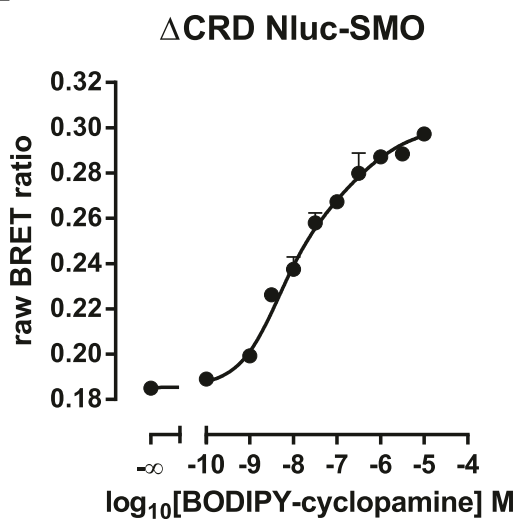

Fig. 2. BODIPY-cyclopamine binding to SMO. (A) Chemical structure of BODIPY-cyclopamine. The BODIPY moiety is highlighted in green. The structure was drawn using ACD/ChemSketch freeware. NanoBRET BODIPY-cyclopamine assays were performed in $\triangle$ SMO HEK293 cells transiently expressing Nluc-tagged SMO [Nluc-SMO; (B and C)] or $\triangle$ CRD Nluc-SMO (D and E). Saturation curves are presented as hyperbolic curves with linear $(\mathrm{B}$ and $\mathrm{D})$ and as sigmoidal curves with logarithmic (C and E) BODIPY-cyclopamine concentrations. Graphs present raw NanoBRET values obtained following 1 hour ligand exposure to living $\Delta$ SMO HEK293 cells. Data points are presented as mean \pm S.E.M. from $n=8$ to 9 individual experiments. Curves for Nluc-SMO were fit to a three-parameter model (log scale) and one-site specific binding (linear scale). For $\Delta$ CRD Nluc-SMO curves were fit according to biphasic (log scale) or two-site specific binding (linear scale).

association at different ligand concentrations using Nluc-SMO and $\triangle$ CRD Nluc-SMO expressing $\Delta$ SMO HEK293 cells (Fig. 3). The kinetic analysis results are summarized in Tables 1 and 2 and Supplemental Fig. 3. The kinetic binding analysis underlined that the affinity of BODIPY-cyclopamine to the $\triangle$ CRD Nluc-SMO was higher and BRET counts were larger compared with Nluc-SMO. The kinetic $K_{\mathrm{d}}$ values $(105 \pm 25$ and $23 \pm 16 \mathrm{nM}$ for the full-length and $\Delta \mathrm{CRD}$ receptors, respectively) were in fair agreement with the values obtained from saturation binding experiments. Moreover, association of BODIPY-cyclopamine $(1000 \mathrm{nM})$ to $\Delta \mathrm{CRD}$ Nluc-SMO followed a two-phase curve arguing for the involvement of another binding site (Table 2), which is consistent with the saturation binding results. To further dissect BODIPY-cyclopamine association to SMO we employed the SMO antagonist SANT-1 at $10 \mu \mathrm{M}$, which interacts with the 7TM core of the receptor (Chen et al., 2002b). For both receptor constructs SANT-1 reduced association of BODIPY-cyclopamine at the lower concentrations, but did not completely abrogate binding of $1000 \mathrm{nM}$ BODIPY-cyclopamine.

NanoBRET-Based Ligand Binding Is Superior to Fluorescence-Based Quantification of BODIPY-cyclopamine Binding to SMO. Cyclopamine is chemically similar to cholesterol, rendering it cell permeable and lipophilic resulting in detectable nonspecific binding to cells and particularly membranes. When comparing the increase in NanoBRET between BODIPY-cyclopamine and Nluc-SMO (or $\Delta$ CRD Nluc-SMO) with the increase in the fluorescence signal emerging from BODIPY-cyclopamine, specific and saturable binding can be detected by NanoBRET already in the lower nanomolar range, especially with the $\triangle \mathrm{CRD}$ Nluc-SMO construct. On the other hand, the nonsaturable increase in fluorescence is detectable only at higher concentrations of BODIPY-cyclopamine. More importantly, the NanoBRET signal saturates at ligand concentrations that produce an unreliable increase in fluorescence, especially in the case of the $\triangle$ CRD Nluc-SMO. At higher BODIPY-cyclopamine concentrations, beyond those required to saturate the NanoBRET signal, a linear increase of fluorescence was detectable, indicating that under these experimental conditions, fluorescence includes a substantial component of unspecific ligand binding (Fig. 4, A and B). This was particularly obvious when comparing the fluorescence signal at a BODIPY-concentration producing maximal binding in the absence and presence of $10 \mu \mathrm{M}$ SANT-1 for the Nluc-SMO and the $\Delta$ CRD NlucSMO constructs (Fig. 4C). At this concentration the NanoBRET signal was blocked by SANT-1, whereas fluorescence was not affected (compare Fig. 3; Fig. 4C). After having 

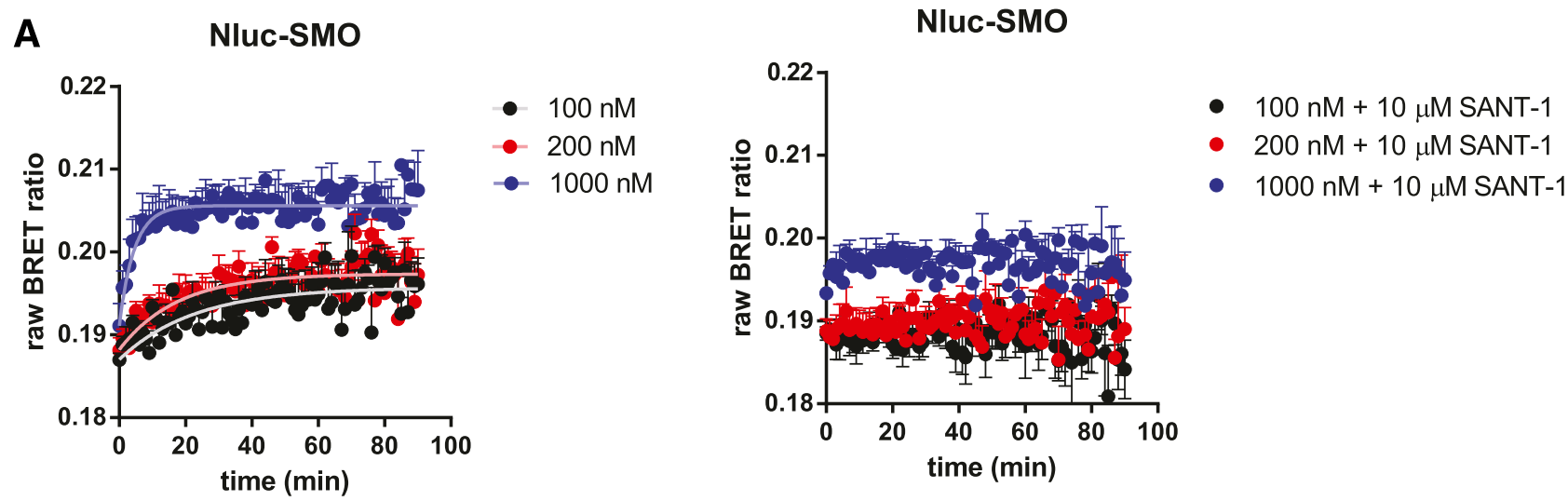

\section{B}
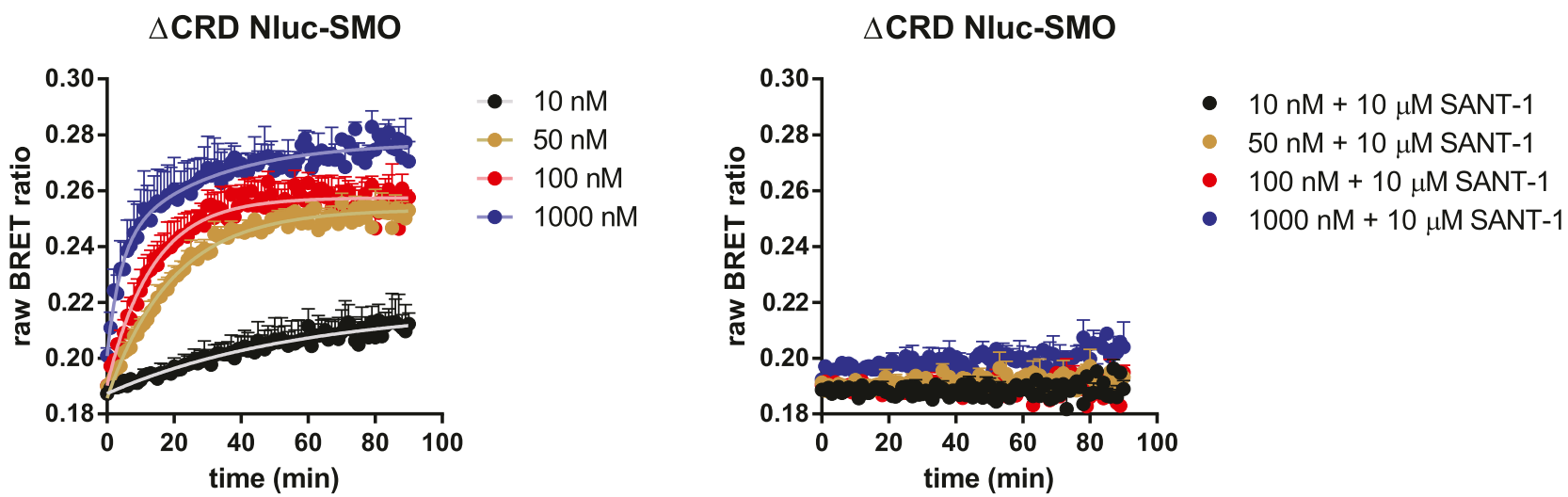

Fig. 3. BODIPY-cyclopamine binding kinetics. Association kinetics of BODIPY-cyclopamine to Nluc-SMO [(A) 100, 200, and 1000 nM BODIPYcyclopamine] or $\triangle$ CRD Nluc-SMO [(B) 10, 50, 100, and $1000 \mathrm{nM}$ BODIPY-cyclopamine] were determined in the absence and presence of the SMO antagonist SANT-1 $(10 \mu \mathrm{M})$ by detection of NanoBRET in living $\Delta$ SMO HEK293 cells over time. NanoBRET was sampled once per minute for 90 minutes. Data points are presented as mean \pm S.E.M. from $n=3$ to 4 individual experiments. Kinetic parameters are summarized in Tables 1 and 2 and Supplemental Fig. 3.

established the superiority of the NanoBRET-based binding assay over fluorescence-based detection of ligand binding, we aimed to investigate if this assay is highthroughput compatible by Z-factor analysis. As expected from the BODIPY-cyclopamine binding parameters, the Z-factor for Nluc-SMO with coelenterazine-h as Nluc substrate was poor comparing basal BODIPY-cyclopamine BRET in the absence and presence of $10 \mu \mathrm{M}$ SANT-1. However, this could be improved by changing to furimazine as Nluc substrate. Furthermore, the Z-factor analysis with the $\triangle$ CRD SMO construct provided an excellent assay window already with coelenterazine-h (Supplemental Fig. 4).

BODIPY-cyclopamine Binding to SMO Is Surmountable. To explore the competitive nature of BODIPYcyclopamine binding to SMO in more detail, we combined BODIPY-cyclopamine with increasing concentrations of

TABLE 1

Kinetic parameters of BODIPY-cyclopamine binding to Nluc-SMO Values are based on data from $n=3$ to 4 individual experiments (shown in Fig. 3A) and shown as a best-fit value \pm S.D.

\begin{tabular}{lcc}
\hline & Nluc-SMO & \\
\hline$k_{\text {on }}$ & $k_{\text {off }}$ & $K_{\mathrm{d}}$ \\
\hline$\left(1 / M^{*} \mathrm{~min}\right) * 10^{5}$ & $l / \min$ & $n M$ \\
$2.00 \pm 0.07$ & $0.021 \pm 0.004$ & $105 \pm 25$ \\
\hline
\end{tabular}

commercially available SMO ligands (Fig. 5A): agonists (purmorphamine and SAG1.3), antagonist (SANT-1), and inverse agonist (cyclopamine-KAAD), employing both the full-length Nluc-SMO (competition with $200 \mathrm{nM}$ BODIPYcyclopamine) as well as the $\triangle$ CRD Nluc-SMO (competition with $10 \mathrm{nM}$ BODIPY-cyclopamine). While cyclopamine-KAAD and SANT-1 presented the highest affinity to Nluc-SMO, the agonist SAG1.3 was intermediate and purmorphamine showed the lowest affinity (Fig. 5B; Table 3). A similar rank order was obtained in the $\triangle \mathrm{CRD}$ Nluc-SMO-transfected cells (Fig. 5C; Table 4). Interestingly, residual BODIPYcyclopamine binding produced NanoBRET, at competitor concentrations sufficiently high to reach saturation, that were substantially higher in the full-length Nluc-SMO compared (Fig. 5B) to $\triangle$ CRD Nluc-SMO (Fig. 5C). At maximal competition SANT-1 reduced BODIPY-cyclopamine $(200 \mathrm{nM})$ binding to $45.2 \%$ of maximal binding, whereas it completely abolished BODIPY-cyclopamine (10 $\mathrm{nM}$ ) binding at the $\triangle \mathrm{CRD}$ NlucSMO ( 0.1\% binding left). These findings indicate that, at the tested concentrations, BODIPY-cyclopamine binding is surmountable to a higher degree at the $\triangle$ CRD Nluc-SMO compared with Nluc-SMO. Additionally, cyclopamine-KAAD competition with BODIPY-cyclopamine at the full-length receptor did not reach the plateau, indicating further displacement of the fluorescent ligand, presumably at a different binding pocket. 
TABLE 2

Kinetic parameters of BODIPY-cyclopamine binding to $\triangle$ CRD Nluc-SMO

Values are based on data from $n=3$ to 4 individual experiments (shown in Fig. 3B) and shown as a best-fit value \pm S.D.

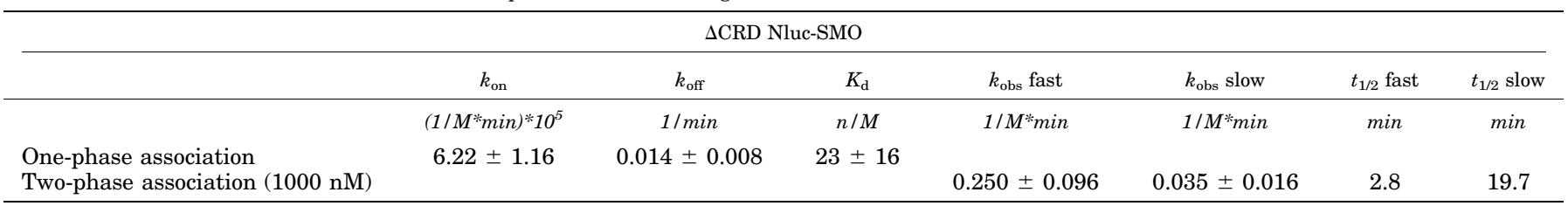

Differential Competition of BODIPY-cyclopamine Binding Allows Pharmacological Separation of Two Binding Sites. The successful targeting of SMO with vismodegib in the therapy of basal cell carcinoma has also led to the discovery of therapy-resistant point mutations in SMO (Zhang et al., 2018a). Here, we introduced the double mutant $\mathrm{D} 477 \mathrm{G}^{6.54} / \mathrm{E}^{2} 22 \mathrm{~K}^{7.38}$ into Nluc-SMO and $\triangle \mathrm{CRD}$ Nluc-SMO (corresponding to human D473 $3^{6.54}$ and E518 ${ }^{7.38}$ mutants) to further dissect the contribution of different binding sites to BODIPY-cyclopamine-SMO interaction. The mutant versions are expressed on the cell surface upon transient transfection in $\triangle$ SMO HEK293 cells (Fig. 6A). To further define the binding characteristics of the two separate BODIPY-cyclopamine binding sites in the 7TM core of the receptor, we made use of a saturating concentration of SANT- $1(10 \mu \mathrm{M})$ and probed the wild-type and $\mathrm{D} 477 \mathrm{G}^{6.54} / \mathrm{E} 522 \mathrm{~K}^{7.38}$ of Nluc-SMO and $\triangle \mathrm{CRD}$ Nluc-SMO with increasing concentrations of BODIPY-cyclopamine (Fig. 6, B and C). In line with the competition data using a fixed BODIPY-cyclopamine concentration, we found that SANT-1, which solely binds to the 7TM ligand binding site of SMO (Wang et al., 2014), reduces the maximal binding of
BODIPY-cyclopamine at the Nluc-SMO by one third with maintained affinity. At the $\triangle$ CRD Nluc-SMO, however, SANT1 virtually prevents BODIPY-cyclopamine interaction with SMO up to a concentration of $100 \mathrm{nM}$. At $100 \mathrm{nM}$ and above, BODIPY-cyclopamine reliably showed saturable, SANT-1 $(10 \mu \mathrm{M})$-insensitive binding in cells transfected with $\Delta \mathrm{CRD}$ Nluc-SMO. The SANT-1-insensitive fraction of BODIPYcyclopamine shows a reduced $B_{\max }$ and a lower affinity. In the full-length Nluc-SMO, the double mutant did not affect $B_{\max }$ of BODIPY-cyclopamine but there was a statistically significant, ca. fourfold decrease in affinity (one-site fit $B_{\max }=0.035,95 \%$ CI [0.032-0.039], $\left.P=0.8193 ; \mathrm{p} K_{\mathrm{d}}=6.2 \pm 0.1 ; P<0.0001\right)$. In $\Delta \mathrm{CRD}$ Nluc-SMO D $477 \mathrm{G}^{6.54} / \mathrm{E} 522 \mathrm{~K}^{7.38}$, the BODIPY-cyclopamine binding followed a one-site curve, as opposed to the wild-type receptor. Furthermore, the affinity was dramatically reduced by ca. 125 -fold ( $\mathrm{p} K_{\mathrm{d}}=6.3 \pm 0.1$ ) and the maximal binding also decreased (one-site fit $B_{\max }=0.074,95 \%$ CI [0.071-0.077], $P<0.0001)$.

In both cases, SANT-1 $(10 \mu \mathrm{M})$, which targets the lower binding pocket, maintains its effect on BODIPY-cyclopamine at both the wild-type and the $\mathrm{D} 477 \mathrm{G}^{6.54} / \mathrm{E} 522 \mathrm{~K}^{7.38}$ for full-length
A

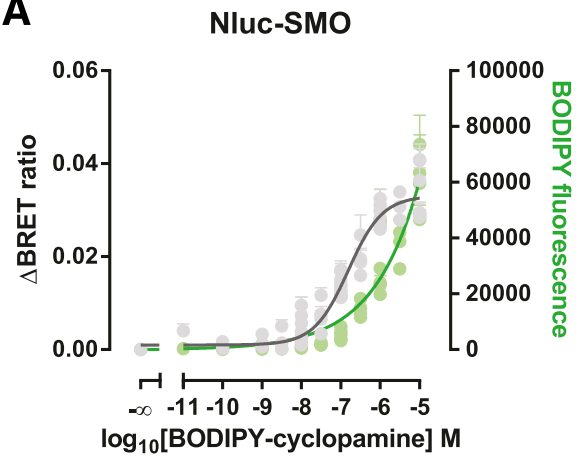

C

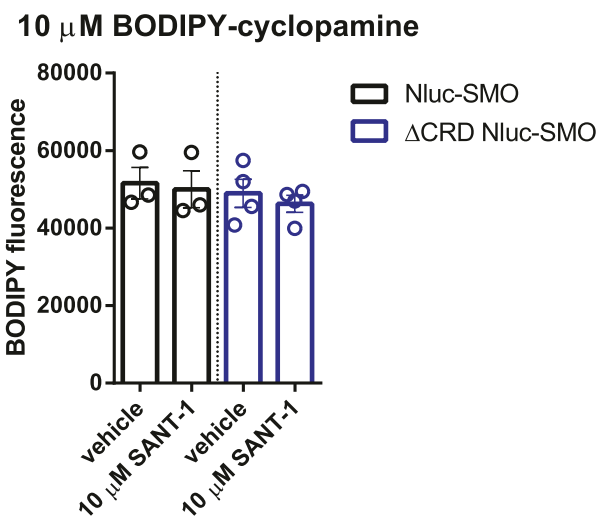

B

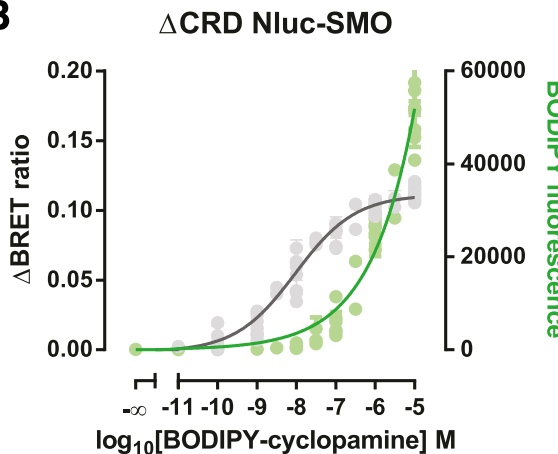

$\log _{10}[$ BODIPY-cyclopamine] M
Fig. 4. Assessment of BODIPY-cyclopamine by NanoBRET is superior to detection of fluorescence. Prior to NanoBRET binding assays with BODIPY-cyclopamine, total fluorescence values (BODIPY) were detected. The graphs present increase in NanoBRET (left axis) and BODIPY fluorescence (right axis; green) for Nluc-SMO (A) and $\triangle$ CRD Nluc-SMO (B). NanoBRET ( $\triangle \mathrm{BRET})$ data are extracted from the experiments shown in Fig. 1. Data points are shown as mean \pm S.D. of each mean value of technical replicates of $n=8$ to 9 individual experiments. Curve fitting for fluorescence values was done using semilog line function in GraphPad Prism 6. (C) BODIPY-cyclopamine fluorescence is compared in the absence and presence of $10 \mu \mathrm{M}$ SANT-1 in experiments with Nluc-SMO or $\triangle$ CRD Nluc-SMO. While SANT-1 dramatically affects NanoBRET readings (see Fig. 3), fluorescence values are not affected. Data present mean \pm S.E.M. from $n=3$ to 4 individual experiments. 
A

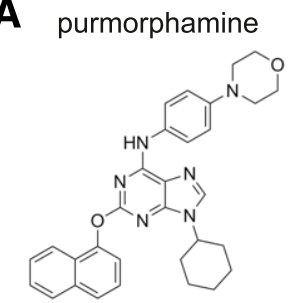

B

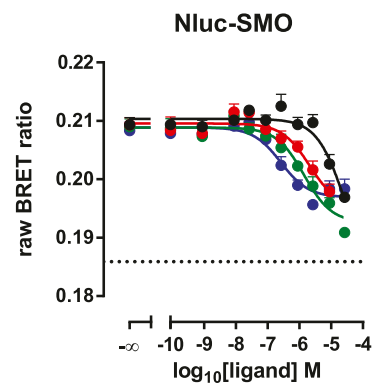

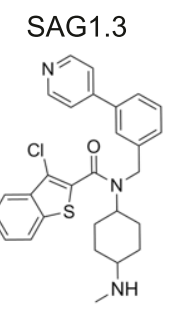

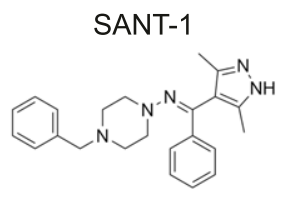

cyclopamine-KAAD

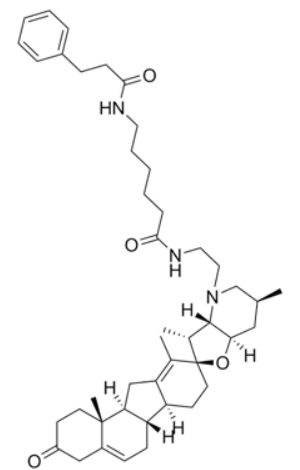

C

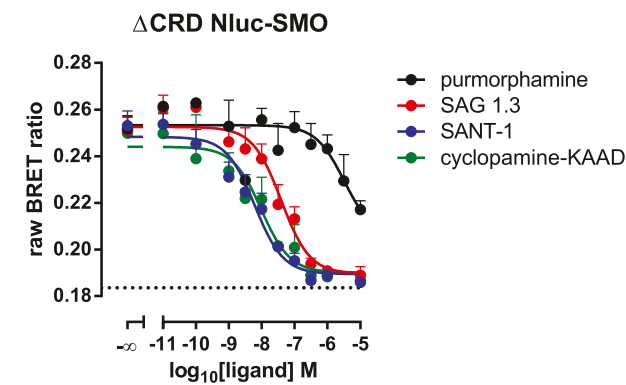

Fig. 5. Competition experiments with BODIPY-cyclopamine and SMO antagonists and agonists. (A) Chemical structures of SMO ligands used in the competition assays. $\triangle$ SMO HEK293 cells expressing Nluc-SMO (B) or $\triangle$ CRD Nluc-SMO (C) were incubated with increasing concentrations of SMO antagonists/inverse agonists (SANT-1, cyclopamine-KAAD) and agonists (SAG1.3, purmorphamine) and subsequently exposed to BODIPY-cyclopamine (200 nM for Nluc-SMO; $10 \mathrm{nM}$ for $\Delta$ CRD Nluc-SMO). Raw NanoBRET data are presented as mean \pm S.E.M. from $n=5$ to 6 individual experiments. Pharmacological parameters are summarized in Tables 3 and 4. Curve fitting was done with a one-site competition binding model. The dotted lines represent raw NanoBRET ratio of the donor-only condition (no BODIPY-cyclopamine added).

and $\triangle$ CRD SMO. Consistently with the SANT-1 binding mode, the double mutant in the upper site does not affect the SANT1-insensitive fraction of BODIPY-cyclopamine binding in $\triangle$ CRD Nluc-SMO (Fig. 6C). In addition, we provide a kinetics analysis of BODIPY-cyclopamine association to D477G 6.54 / E522K ${ }^{7.38} \Delta$ CRD Nluc-SMO (Fig. 6D; Table 5). The association of BODIPY-cyclopamine to $\triangle \mathrm{CRD}$ Nluc-SMO D477G $\mathrm{G}^{6.54}$ / E522K ${ }^{7.38}$ follows a one-phase association curve with a "kinetic" $K_{\mathrm{d}}=1403 \pm 701 \mathrm{nM}$, which is in fair agreement (ca. threefold decrease) with the saturation binding data.

Molecular Docking of BODIPY-cyclopamine Supports the Two-binding Site Model. To obtain more detailed insights into the BODIPY-cyclopamine binding at the atomistic level, we set up a molecular docking study. We selected the recent SMO structure [PDB ID: 6O3C (Deshpande et al., 2019)] as a target for our docking, as it

\section{TABLE 3}

Binding affinities of various SMO ligands in competition with BODIPYcyclopamine binding $(200 \mathrm{nM})$ to Nluc-SMO

Data are based on $n=5$ individual experiments presented in Fig. 5B. p $K_{\mathrm{i}}$ values are presented as a best-fit value \pm S.D.

\begin{tabular}{lc}
\hline \multicolumn{2}{c}{ Nluc-SMO } \\
\hline Ligand & $\mathrm{p} K_{\mathrm{i}} \pm$ S.D. vs. $200 \mathrm{nM}$ BODIPY-cyclopamine \\
\hline SANT-1 & $6.9 \pm 0.2$ \\
Cyclopamine-KAAD & $6.2 \pm 0.1$ \\
SAG1.3 & $6.0 \pm 0.2$ \\
Purmorphamine & $4.9 \pm 0.3$ \\
\hline
\end{tabular}

manifests the two 7TM binding sites (i.e., they are occupied with small molecular ligands; Fig. 7A). At the upper binding pocket, BODIPY-cyclopamine occupies hook-like conformations, wherein the cyclopamine-moiety of the molecule is buried within the 7TM core of the receptor and the BODIPYmoiety is exposed to the solvent. The main polar interactions at the upper pocket are with $\mathrm{E} 518^{7.38}$ (note: the crystal structure is of the human SMO) and K395 at ECL2. At the lower binding pocket, the whole BODIPY-cyclopamine molecule is bound within the 7TM core of the receptor, the main polar interaction counterparts being with T528 ${ }^{7.48}$, E518 ${ }^{7.38}$, and N219 at the N terminus. Furthermore, in silico analysis of solvent-accessible surfaces revealed a better ligandaccessibility of $\triangle \mathrm{CRD}$ SMO compared with the wild-type receptor (Fig. 7B) as expected from the difference in binding parameters between full-length and $\triangle \mathrm{CRD}$ SMO.

\section{TABLE 4}

Binding affinities of various SMO ligands in competition with BODIPYcyclopamine binding $(10 \mathrm{nM})$ to $\triangle \mathrm{CRD}$ Nluc-SMO

Data are based on $n=5$ to 6 individual experiments presented in Fig. 5C. p $K_{\mathrm{i}}$ values are presented as a best-fit value \pm S.D.

\begin{tabular}{lc}
\hline \multicolumn{2}{c}{$\Delta$ CRD Nluc-SMO } \\
\hline Ligand & $\mathrm{p} K_{\mathrm{i}} \pm$ S.D. vs. $10 \mathrm{nM}$ BODIPY-cyclopamine \\
\hline SANT-1 & $8.8 \pm 0.1$ \\
Cyclopamine-KAAD & $8.6 \pm 0.2$ \\
SAG1.3 & $8.0 \pm 0.1$ \\
Purmorphamine & $6.1 \pm 0.4$ \\
\hline
\end{tabular}


A

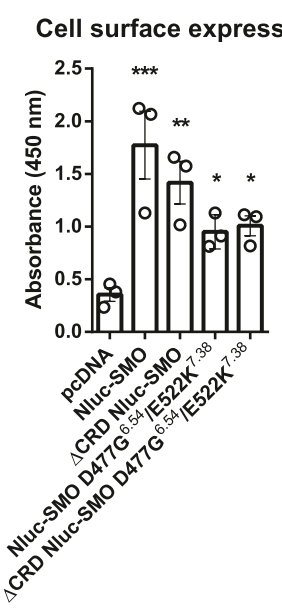

B

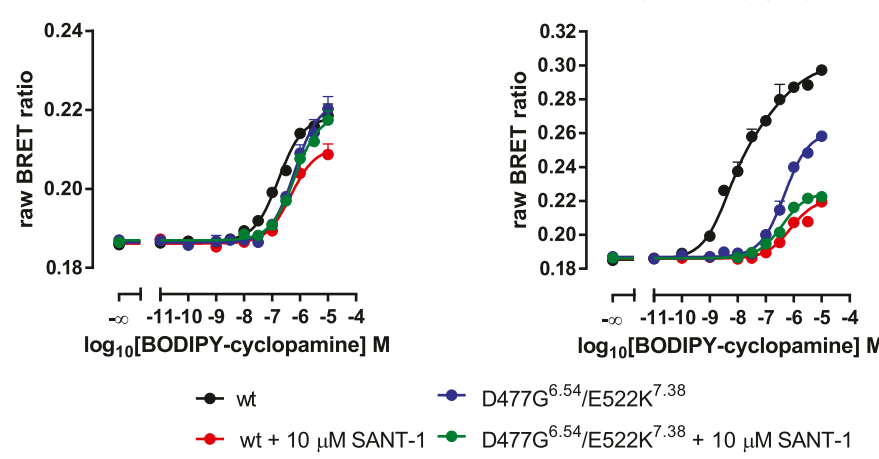

D

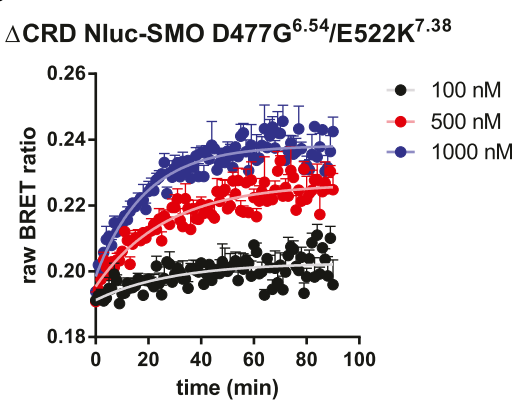

\section{Discussion}

The development of a NanoBRET-based ligand binding assay provides an interesting complement to GPCR pharmacology, enabling ligand binding studies on living cells in real time with simplified protocols (Stoddart et al., 2015, 2018a). Here, we optimize this assay for the Class F receptor SMO improving sensitivity and performance of previously used fluorescence-based approaches (Chen et al., 2002a,b; Manetti et al., 2010; Tao et al., 2011; Gorojankina et al., 2013; Huang et al., 2016, 2018). Due to its large assay window for specific binding and the low influence of unspecific binding this NanoBRET-based assay is particularly suitable for lipophilic ligands such as cyclopamine and potentially other cholesterol-like molecules, which target SMO and generally show unspecific interactions with the membrane. Furthermore, this high-throughput compatible assay should be adaptable to any fluorescently tagged molecule acting as SMO ligand and could, provided small molecules become available to target Frizzleds, also be employed for other Class $\mathrm{F}$ receptors.

TABLE 5

Kinetic parameters of BODIPY-cyclopamine binding to $\triangle$ CRD Nluc-SMO $\mathrm{D} 477 \mathrm{G}^{6.54} / \mathrm{E} 522 \mathrm{~K}^{7.38}$

Values are based on data from $n=3$ individual experiments (shown in Fig. 6D) and shown as a best-fit value \pm S.D.

\begin{tabular}{lcc}
\hline \multicolumn{3}{c}{$\Delta$ CRD Nluc-SMO D $477 \mathrm{G}^{6.54} / \mathrm{E} 522 \mathrm{~K}^{7.38}$} \\
\hline$k_{\text {on }}$ & $k_{\text {off }}$ & $K_{\mathrm{d}}$ \\
\hline $1 / M^{*} \mathrm{~min}^{*} 10^{5}$ & $1 / \min$ & $n M$ \\
$0.22 \pm 0.07$ & $0.030 \pm 0.005$ & $1403 \pm 701$ \\
\hline
\end{tabular}

Fig. 6. Combining BODIPY-cyclopamine with SANT-1 allows pharmacological dissection of two separate binding sites in the 7TM core of SMO. (A) Surface ELISA was used to assess cell surface expression of wild-type and $\mathrm{D} 477 \mathrm{G}^{6.54}$ / E522 $\mathrm{K}^{7.38}$ double mutant of Nluc-SMO and $\triangle$ CRD Nluc-SMO upon overexpression in $\triangle$ SMO HEK293 cells. Raw data are shown from $n=3$ individual experiments and are presented as mean $\pm \mathrm{SEM} ; * P<0.05, * * P<0.01$, $* * * P<0.001$ (one-way ANOVA). BODIPY-cyclopamine binding curves were determined in $\triangle$ SMO HEK293 cells expressing either Nluc-SMO (B) or $\triangle$ CRD Nluc-SMO (C) either in the absence or presence of a saturating concentration of the SMO antagonist SANT-1 $(10 \mu \mathrm{M})$, which targets the lower pocket of the 7TM ligand binding site of SMO. Data points are presented as mean \pm S.E.M. from $n=3-5$ individual experiments. Values for the wild-type SMO in the absence of SANT-1 are from Fig. 2C (Nluc-SMO) and Fig. 2E ( $\triangle \mathrm{CRD}$ Nluc-SMO). (D) Association kinetics of BODIPY-cyclopamine $(100,500,1000 \mathrm{nM})$ to $\triangle$ CRD Nluc-SMO $\mathrm{D} 477 \mathrm{G}^{6.54} / \mathrm{E}^{2} 22 \mathrm{~K}^{7.38}$. Data from $n=3$ individual experiments are presented as mean \pm S.E.M. Kinetic parameters are summarized in Table 5 and Supplemental Fig. 3.
Recent insight into the molecular mechanisms of drug action on SMO by crystallography and CryoEM provide somewhat controversial yet intriguing information regarding cyclopamine and cholesterol interaction with the 7TM ligandbinding site and the CRD (Weierstall et al., 2014; Huang et al., 2016, 2018; Deshpande et al., 2019; Qi et al., 2019). Here, we have been able to pharmacologically separate two BODIPYcyclopamine binding sites on the 7TM core of SMO. It has been reported that total BODIPY-cyclopamine binding to fulllength SMO is composed of at least two components: ligand binding to the CRD (Huang et al., 2016, 2018), for which we did not find evidence for in our experiments, and the 7TM core (Weierstall et al., 2014; Huang et al., 2018). Most importantly, SANT-1, which solely binds to the receptor core in the lower pocket of the SMO binding site (Wang et al., 2014), competes with BODIPY-cyclopamine more efficiently in the $\triangle$ CRD NlucSMO compared with the full length receptor. This large increase in affinity and in NanoBRET signal $\left(B_{\max }\right)$, suggests that the CRD exerts a negative allosteric modulation on BODIPY-cyclopamine binding to the SMO 7TM core. The residual saturable binding of BODIPY-cyclopamine to $\triangle \mathrm{CRD}$ Nluc-SMO above $10^{-7} \mathrm{M}$ identifies a SANT-1-insensitive fraction, suggesting an additional binding pocket for BODIPYcyclopamine. While BODIPY-cyclopamine binding to $\triangle \mathrm{CRD}$ Nluc-SMO clearly follows a two-site (biphasic) regression fit, the ligand coupling to the full-length receptor follows a typical one-site model. Therefore, we assume that this SANT-1-insensitive binding site cannot be solely explained by a potential non-specific BODIPY-cyclopamine association to membranes as this second pocket would also become more apparent at the full-length receptor. Moreover, non-specific 

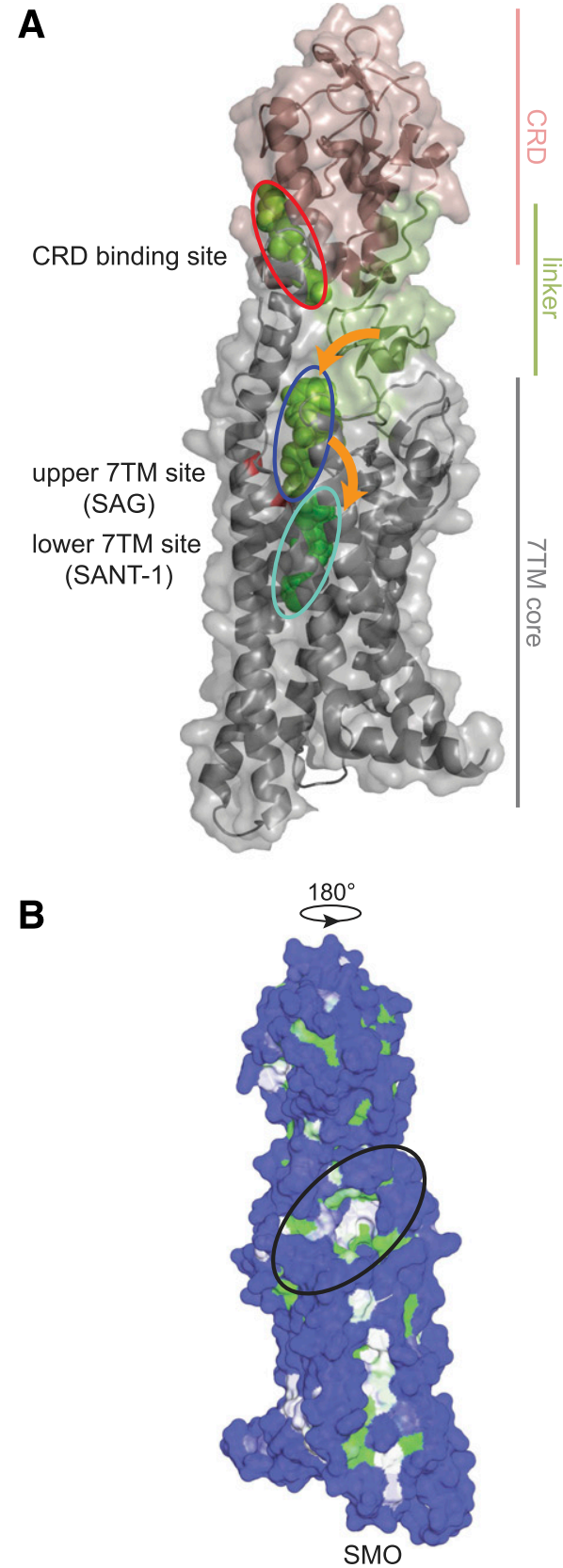
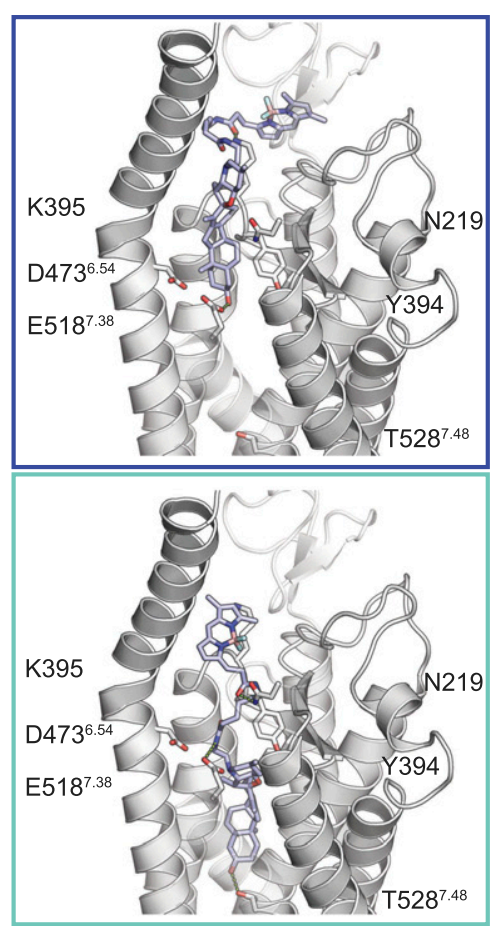

Fig. 7. Separate ligand binding sites on SMO. (A) Left panel: schematic presentation of reported SMO ligand binding sites. The receptor representation (transparent surface view with protein backbone shown as ribbon) was derived from the SMO crystal structure bound to Nanobody NbSmo8 and SAG21k (PDB ID: 6O3C) (Deshpande et al., 2019). The major subdomains (CRD, linker, and 7TM core) are color-coded. Ligands are shown as spheres and are highlighted in green. Reported ligand binding sites on the CRD and in the 7TM core are encircled in red, blue, and light blue, respectively. While our data did not indicate BODIPY-cyclopamine binding to the CRD site, the NanoBRET-based binding assay supports two communicating binding sites for BODIPY-cyclopamine in the 7TM core of SMO. The mutations D $477 \mathrm{G}^{6.54} / \mathrm{E} 522 \mathrm{~K}^{7.38}$ in TM6 and TM7, which affect BODIPY-cyclopamine binding to SMO are depicted in red. Based on the effects of D $477 \mathrm{G}^{6.54} / \mathrm{E} 522 \mathrm{~K}^{7.38}$ and SANT-1 on BODIPY-cyclopamine binding we suggest a twostep binding mode of BODIPY-cyclopamine involving the upper and the lower 7TM site (orange arrows). Right panel: docking of BODIPYcyclopamine showed that it indeed could occupy either only the binding site of SAG (top; suggested low-affinity binding site; blue frame) or the binding sites of both SAG and SANT-1 (bottom; suggested high-affinity binding site; light blue frame). The highest-scoring BODIPY-cyclopamine poses (violet sticks) are shown (according to the Glide DockingScore). The receptor is presented as a white cartoon and amino acid residues suggested to contribute to ligand binding as white sticks. The view is corresponding to that of the left panel. (B) Solvent-accessible surfaces (SASs) of SMO (left; PDB ID: $603 C$ ) and $\triangle$ CRD-SMO (right; PDB ID: 6OT0) indicate that the 7TM binding site of $\triangle \mathrm{CRD}$ $\mathrm{SMO}$ is more accessible for ligand binding than that of the SMO. The surface is colored by the solventaccessibility of each residue; blue = "exposed," over $25 \%$ of maximum SAS; green = "buried," less than $10 \%$ of maximum SAS; white = residues with SASvalues between these two. The view is from behind the TM4 and TM5, and the opening to the binding funnel is encircled in black. The surface corresponding to amino acids 388-397 is hidden to get better view. (A) was produced in PyMOL (The PyMOL Molecular Graphics System, Version 2.0; Schrödinger, LLC), and (B) in Biovia DiscoveryStudio Visualizer 2017 R2 (Dassault Systèmes SE).

binding would most likely increase linearly. Given the simultaneous binding of SAG21k and cholesterol to SMO in the recent crystal structure (PDB ID: 6O3C) of active, nanobody NbSmo8-bound SMO (Deshpande et al., 2019) and the fact that SANT-1 and cyclopamine occupy two different parts of the small molecule binding space in SMO (Wang et al., 2014; Weierstall et al., 2014; Huang et al., 2018) it could also be possible that BODIPY-cyclopamine and SANT1 bind the 7TM core simultaneously. It cannot be excluded that the binding modes of BODIPY-cyclopamine and cyclopamine are different as it remains to be verified by structural studies. Importantly, a recent study on ALLO-1, a small molecule ligand targeting the lower pocket of SMO, showed that this compound competes with BODIPY-cyclopamine but not with $\left[{ }^{3} \mathrm{H}\right]$ cyclopamine, further supporting our two sites hypothesis (Zhou et al., 2019). Along these lines, distinct binding poses of cyclopamine and related sterols were reported in several crystal structures using SMO from various species. In summary, there is a cyclopamine/sterol binding site in the lipid groove of the CRD (Byrne et al., 2016; Huang et al., 2016, 2018; Deshpande et al., 2019), one in the upper pocket of the 7TM core (Huang et al., 2018; Qi et al., 2019) and one in the lower position (Deshpande et al., 2019), which overlaps with the SANT-1 binding pocket (Wang et al., 2014) (Fig. 7A). Moreover, it has been demonstrated that SAG1.3-induced fulllength SMO-mediated Gli transcriptional activity still reaches saturation, albeit at lower efficacy following treatment with SANT-1.This further provides functional evidence that both the upper (SAG binding site) and the lower pocket (SANT-1 binding site) in the 7TM core of SMO can be occupied by ligands simultaneously and that these pockets may allosterically regulate each other (Chen et al., 2002b). Similar 
conclusions were drawn from radioligand binding studies (Frank-Kamenetsky et al., 2002; Tao et al., 2011). Furthermore, the phenomenon of allosteric regulation in the SMO binding site was also inferred from studies on other SMO ligands (Hoch et al., 2015; Chen et al., 2016).

Interestingly, removal of the CRD of SMO increased both $B_{\max }$ and the $K_{\mathrm{d}}$ of BODIPY-cyclopamine. The absence of the CRD, which obviously includes removal of the proposed CRD binding site for BODIPY-cyclopamine, most likely provides better access to the normally buried binding site (the lower binding pocket) in the core of the receptor causing a left shift and an increased $B_{\max }$ (Fig. 7B). Related to that, one might also postulate an allosteric action of the CRD on the ligandbinding sites on the 7TM core, an idea that is fueled by the observation that $\triangle$ CRD SMO exerts a higher constitutive activity (Byrne et al., 2016; Raleigh et al., 2018). A part of the explanation for the efficacy shift of the BODIPY-cyclopamine binding curve could also be the altered BRET parameters, because the distance of the NanoBRET donor and the acceptor could be shorter in the $\triangle$ CRD SMO compared with the fulllength receptor. However, while the different BRET efficiencies in the two receptor constructs would affect the amplitude of the NanoBRET signal originating from BODIPYcyclopamine binding, they cannot explain the leftward shift of the binding curves, indicating a higher affinity to the $\triangle \mathrm{CRD}$ Nluc-SMO. It should be noted that in previous publications low concentrations of BODIPY-cyclopamine (usually $5 \mathrm{nM}$ ) were used for SMO binding assays. Given our data regarding the different affinities of the separate BODIPY-cyclopamine binding sites, these low ligand concentrations neither allowed sampling the SANT-1-insensitive, low affinity site (the upper binding pocket) in the core of $\triangle \mathrm{CRD}-\mathrm{Nluc}$ SMO nor the putative site on the CRD of the full-length receptor. Application of the novel NanoBRET methodology, however, allows to reliably detect even picomolar and nanomolar amounts of BODIPY-cyclopamine bound to $\triangle \mathrm{CRD}$ Nluc-SMO and $\triangle \mathrm{CRD}$ Nluc-SMO D477G $\mathrm{G}^{6.54} / \mathrm{E} 522 \mathrm{~K}^{7.38}$.

In summary, we dissect BODIPY-cyclopamine interaction with two allosterically linked binding sites on the SMO 7TM core with different affinities. Allosteric interaction between these pockets and BODIPY-cyclopamine binding to the lower one (the high affinity site) can also be inferred from the mutagenesis results reported recently (Deshpande et al., 2019). Here, we propose that the BODIPY-cyclopamine highaffinity site is the deep SANT-1 binding pocket (the lower binding pocket). Since the $\mathrm{D} 477 \mathrm{G}^{6.54} / \mathrm{E} 522 \mathrm{~K}^{7.38}$ in TM6 and TM7 partially affect the high-affinity component of BODIPYcyclopamine binding, it could be that interaction with $\mathrm{D} 477^{6.54} / \mathrm{E} 522^{7.38}$ provides a transition mechanism of ligand binding to the deeper pocket (the high affinity site). Lowaffinity binding to the $\mathrm{D} 477 \mathrm{G}^{6.54} / \mathrm{E} 522 \mathrm{~K}^{7.38}$ mutant in the upper 7TM site (the low affinity site) is still possible and that binding is insensitive to allosteric modulation by SANT-1 binding to the deeper site (the high affinity site). Interestingly, the difference in $B_{\max }$ between the wild-type and mutated $\Delta$ CRD Nluc-SMO [ $\Delta$ CRD Nluc-SMO $B_{\max 1}+\Delta$ CRD NlucSMO $B_{\max 2}$ ) $-\Delta$ CRD Nluc-SMO D $477 \mathrm{G}^{6.54} / \mathrm{E}^{2} 22 \mathrm{~K}^{7.38} B_{\max }$ $\sim 0.039$ ] corresponds well to the $\Delta$ CRD Nluc-SMO $B_{\max 2}$ $(\sim 0.033)$ and Nluc-SMO $B_{\max }(\sim 0.034)$. Assuming no alterations in BRET transfer efficiency between different constructs, it could be that binding of BODIPY-cyclopamine to the upper pocket (the low affinity site) of a ligand-free Nluc-SMO is virtually nondetectable. Moreover, BODIPY-cyclopamine/ SMO interactions in living cells surely depend also on the conformational states of the receptor as well as the presence of endogenous SMO ligand - cholesterol. These factors further add to the complexity of this binding mechanism.

It needs to be noted that the predicted conformational space of BODIPY-cyclopamine is large; one has to be careful when interpreting the docking results. However, the in silico studies indicate that the ligand can interact with E518 ${ }^{7.38}$ (corresponds to E522 $2^{7.38}$ in mouse SMO) in both binding pockets. In the upper pocket (the low-affinity site), the interaction partner is a hydroxyl group of BODIPY-cyclopamine, which can also interact with $\mathrm{K}$ in the mutated receptor. In the lower pocket (the high-affinity site), the partner is an amide nitrogen that cannot interact with $\mathrm{K}$, however, the adjacent oxygen could. In both cases, $D \rightarrow G^{6.54}$ renders the binding site more spacious. As a consequence, different ligand poses could be obtained compared with the wild-type SMO. Furthermore, the docking scores are in line with our two binding site model hypotheses; the average scores of all the high affinity and the low affinity sites poses are $-8.3 \pm 2.4(17$ poses $)$ and $-4.3 \pm 1.7$ (602 poses), respectively (Supplemental Fig. 5). In addition to the allosteric interaction between the two binding pockets, BODIPY-cyclopamine binding, with its cyclopamine core and the linker-BODIPY moiety occupying the lower and the upper pockets, respectively, would also be in line with the competition binding data showing that the ligands interacting with either pocket could, at least partially, displace this fluorescent ligand.

For drug discovery efforts, the $\triangle$ CRD Nluc-SMO probe presents a valuable tool with an advantageous assay window. The intrinsic caveat of the lack of the physiologically relevant CRD, however, remains, requiring thorough validation of screening hits in assays relying on full length SMO. Further work will address in which way the separate ligand-binding sites interact allosterically and what role the CRD-core contacts play for that potential communication.

\section{Acknowledgments}

We thank Stephen Hill (University of Nottingham, UK) for the Nluc- $\mathrm{A}_{3}$ construct and Anna Krook (Karolinska Institutet, Sweden) for the access to the CLARIOstar plate reader.

\section{Authorship Contributions}

Participated in research design: Kozielewicz, Schulte.

Conducted experiments: Kozielewicz, Bowin, Turku.

Contributed new analytic tools: Kozielewicz, Bowin.

Performed data analysis: Kozielewicz, Bowin, Turku, Schulte.

Wrote or contributed to the writing of the manuscript: Kozielewicz, Bowin, Turku, Schulte.

\section{References}

Ballesteros JA and Weinstein H (1995) Integrated methods for the construction of three-dimensional models and computational probing of structure-function relations in G protein-coupled receptors. Methods Neurosci 25:366-428.

Bee WT, Xie W, Truong M, Will M, Turunen B, Zuercher WJ, McMillan L, Li H, Hornberger KR, Davenport EA, et al. (2012) The development of a high-content screening binding assay for the smoothened receptor. J Biomol Screen 17:900-911.

Bosma R, Stoddart LA, Georgi V, Bouzo-Lorenzo M, Bushby N, Inkoom L, Waring MJ, Briddon SJ, Vischer HF, Sheppard RJ, et al. (2019) Probe dependency in the determination of ligand binding kinetics at a prototypical $\mathrm{G}$ protein-coupled receptor. Sci Rep 9:7906.

Bouzo-Lorenzo M, Stoddart LA, Xia L, IJzerman AP, Heitman LH, Briddon SJ, and Hill SJ (2019) A live cell NanoBRET binding assay allows the study of ligandbinding kinetics to the adenosine $A_{3}$ receptor. Purinergic Signal 15:139-153.

Byrne EFX, Sircar R, Miller PS, Hedger G, Luchetti G, Nachtergaele S, Tully MD, Mydock-McGrane L, Covey DF, Rambo RP, et al. (2016) Structural basis of Smoothened regulation by its extracellular domains. Nature 535:517-522. 
Chen B, Trang V, Lee A, Williams NS, Wilson AN, Epstein EH Jr, Tang JY, and Kim $\mathrm{J}$ (2016) Posaconazole, a second-generation triazole antifungal drug, inhibits the hedgehog signaling pathway and progression of basal cell carcinoma. Mol Cancer Ther 15:866-876.

Chen JK (2016) I only have eye for ewe: the discovery of cyclopamine and development of Hedgehog pathway-targeting drugs. Nat Prod Rep 33:595-601.

Chen JK, Taipale J, Cooper MK, and Beachy PA (2002a) Inhibition of Hedgehog signaling by direct binding of cyclopamine to Smoothened. Genes Dev 16: $2743-2748$

Chen JK, Taipale J, Young KE, Maiti T, and Beachy PA (2002b) Small molecule modulation of Smoothened activity. Proc Natl Acad Sci USA 99:14071-14076.

Cheng Y and Prusuff WH (1973) Relationship between the inhibition constant (K1) and the concentration of inhibitor which causes 50 per cent inhibition (I50) of an enzymatic reaction. Biochem Pharmacol 22:3099-3108.

Deshpande I, Liang J, Hedeen D, Roberts KJ, Zhang Y, Ha B, Latorraca NR, Faust B, Dror RO, Beachy PA, et al. (2019) Smoothened stimulation by membrane sterols drives Hedgehog pathway activity. Nature 571:284-288.

Frank-Kamenetsky M, Zhang XM, Bottega S, Guicherit O, Wichterle H, Dudek H, Bumcrot D, Wang FY, Jones S, Shulok J, et al. (2002) Small-molecule modulators of Hedgehog signaling: identification and characterization of Smoothened agonists and antagonists. $J$ Biol 1:10.

Gorojankina T, Hoch L, Faure H, Roudaut H, Traiffort E, Schoenfelder A, Girard N, Mann A, Manetti F, Solinas A, et al. (2013) Discovery, molecular and pharmacological characterization of GSA-10, a novel small-molecule positive modulator of Smoothened. Mol Pharmacol 83:1020-1029.

Hoch L, Faure H, Roudaut H, Schoenfelder A, Mann A, Girard N, Bihannic L, Ayrault O, Petricci E, Taddei M, et al. (2015) MRT-92 inhibits Hedgehog signaling by blocking overlapping binding sites in the transmembrane domain of the Smoothened receptor. FASEB J 29:1817-1829.

Hoy SM (2019) Glasdegib: first global approval. Drugs 79:207-213.

Huang P, Nedelcu D, Watanabe M, Jao C, Kim Y, Liu J, and Salic A (2016) Cellular cholesterol directly activates smoothened in hedgehog signaling. Cell 166:1176-1187.

Huang P, Zheng S, Wierbowski BM, Kim Y, Nedelcu D, Aravena L, Liu J, Kruse AC, and Salic A (2018) Structural basis of smoothened activation in hedgehog signaling [published correction appears in Cell (2018) 175:295-297]. Cell 174:312-324.e16.

Incardona JP, Gaffield W, Kapur RP, and Roelink H (1998) The teratogenic Veratrum alkaloid cyclopamine inhibits sonic hedgehog signal transduction. Development 125:3553-3562.

Ingham PW and McMahon AP (2001) Hedgehog signaling in animal development: paradigms and principles. Genes Dev 15:3059-3087.

Kong JH, Siebold C, and Rohatgi R (2019) Biochemical mechanisms of vertebrate hedgehog signaling. Development 146:dev166892.

Kowatsch C, Woolley RE, Kinnebrew M, Rohatoi R, and Siebold C (2019) Structures of vertebrate Patched and Smoothened reveal intimate links between cholesterol and Hedgehog signalling. Curr Opin Struct Biol 57:204-214.

Lu W, Zhang D, Ma H, Tian S, Zheng J, Wang Q, Luo L, and Zhang X (2018) Discovery of potent and novel smoothened antagonists via structure-based virtual screening and biological assays. Eur J Med Chem 155:34-48.

Manetti F, Faure H, Roudaut H, Gorojankina T, Traiffort E, Schoenfelder A, Mann A, Solinas A, Taddei M, and Ruat M (2010) Virtual screening-based discovery and mechanistic characterization of the acylthiourea MRT-10 family as smoothened antagonists. Mol Pharmacol 78:658-665.

Mocking TAM, Verweij EWE, Vischer HF, and Leurs R (2018) Homogeneous, realtime NanoBRET binding assays for the histamine $\mathrm{H}_{3}$ and $\mathrm{H}_{4}$ receptors on living cells. Mol Pharmacol 94:1371-1381.

Nachtergaele S, Mydock LK, Krishnan K, Rammohan J, Schlesinger PH, Covey DF, and Rohatgi R (2012) Oxysterols are allosteric activators of the oncoprotein Smoothened. Nat Chem Biol 8:211-220.

Qi X, Liu H, Thompson B, McDonald J, Zhang C, and Li X (2019) Cryo-EM structure of oxysterol-bound human Smoothened coupled to a heterotrimeric $\mathrm{G}_{\mathrm{i}}$. Nature 571: 279-283.

Raleigh DR, Sever N, Choksi PK, Sigg MA, Hines KM, Thompson BM, Elnatan D, Jaishankar P, Bisignano P, Garcia-Gonzalo FR, et al. (2018) Cilia-associated oxysterols activate smoothened. Mol Cell 72:316-327.
Rominger CM, Bee WL, Copeland RA, Davenport EA, Gilmartin A, Gontarek R, Hornberger KR, Kallal LA, Lai Z, Lawrie K et al. (2009) Evidence for allosteric interactions of antagonist binding to the smoothened receptor. J Pharmacol Exp Ther 329:995-1005.

Schulte G (2010) International Union of Basic and Clinical Pharmacology. LXXX. The class Frizzled receptors. Pharmacol Rev 62:632-667.

Schulte G and Kozielewicz P (2019) Structural insight into Class F receptors - what have we learnt regarding agonist-induced activation? Basic Clin Pharmacol Toxicol DOI: $10.1111 /$ bcpt.13235 [published ahead of print]

Sever N, Mann RK, Xu L, Snell WJ, Hernandez-Lara CI, Porter NA, and Beachy PA (2016) Endogenous B-ring oxysterols inhibit the Hedgehog component Smoothened in a manner distinct from cyclopamine or side-chain oxysterols. Proc Natl Acad Sci USA 113:5904-5909.

Shelley JC, Cholleti A, Frye LL, Greenwood JR, Timlin MR, and Uchimaya M (2007) Epik: a software program for $\mathrm{pK}$ ( a ) prediction and protonation state generation for drug-like molecules. J Comput Aided Mol Des 21:681-691.

Stoddart LA, Johnstone EKM, Wheal AJ, Goulding J, Robers MB, Machleidt T, Wood KV, Hill SJ, and Pfleger KDG (2015) Application of BRET to monitor ligand binding to GPCRs. Nat Methods 12:661-663.

Stoddart LA, Kilpatrick LE, and Hill SJ (2018a) NanoBRET approaches to study ligand binding to GPCRs and RTKs. Trends Pharmacol Sci 39:136-147.

Stoddart LA, Vernall AJ, Bouzo-Lorenzo M, Bosma R, Kooistra AJ, de Graaf C, Vischer HF, Leurs R, Briddon SJ, Kellam B, et al. (2018b) Development of novel fluorescent histamine $\mathrm{H}_{1}$-receptor antagonists to study ligand-binding kinetics in living cells. Sci Rep 8:1572.

Sykes DA, Stoddart LA, Kilpatrick LE, and Hill SJ (2019) Binding kinetics of ligands acting at GPCRs. Mol Cell Endocrinol 485:9-19.

Taipale J, Chen JK, Cooper MK, Wang B, Mann RK, Milenkovic L, Scott MP, and Beachy PA (2000) Effects of oncogenic mutations in Smoothened and Patched can be reversed by cyclopamine. Nature 406:1005-1009.

Tao H, Jin Q, Koo DI, Liao X, Englund NP, Wang Y, Ramamurthy A, Schultz PG, Dorsch M, Kelleher J, et al. (2011) Small molecule antagonists in distinct binding modes inhibit drug-resistant mutant of smoothened. Chem Biol 18:432-437.

Wang C, Wu H, Evron T, Vardy E, Han GW, Huang XP, Hufeisen SJ, Mangano TJ, Urban DJ, Katritch V, et al. (2014) Structural basis for Smoothened receptor modulation and chemoresistance to anticancer drugs. Nat Commun 5:4355.

Weierstall U, James D, Wang C, White TA, Wang D, Liu W, Spence JC, Bruce Doak R, Nelson G, Fromme P, et al. (2014) Lipidic cubic phase injector facilitates membrane protein serial femtosecond crystallography. Nat Commun 5:3309.

Wright SC, Kozielewicz P, Kowalski-Jahn M, Petersen J, Bowin CF, Slodkowicz G, Solano-Marti M, Rodriguez D, Hot B, Okashah N, et al. (2019) A conserved molecular switch in Class $\mathrm{F}$ receptors regulates receptor activation and pathway selection. Nature Communications 10, doi: 10.1038/s41467-019-08630-2.

Wu F, Zhang Y, Sun B, McMahon AP, and Wang Y (2017) Hedgehog signaling: from basic biology to cancer therapy. Cell Chem Biol 24:252-280.

Zhang H, Sun Z, Liu Z, and Song C (2018a) Overcoming the emerging drug resistance of smoothened: an overview of small-molecule SMO antagonists with antiresistance activity. Future Med Chem 10:2855-2875.

Zhang JH, Chung TD, and Oldenburg KR (1999) A simple statistical parameter for use in evaluation and validation of high throughput screening assays. J Biomol Screen 4:67-73.

Zhang Y, Bulkley DP, Xin Y, Roberts KJ, Asarnow DE, Sharma A, Myers BR, Cho W, Cheng Y, and Beachy PA (2018b) Structural basis for cholesterol transport-like activity of the hedgehog receptor patched. Cell 175:1352-1364.

Zhou F, Ding K, Zhou Y, Liu Y, Liu X, Zhao F, Wu Y, Zhang X, Tan Q, Xu F, et al. (2019) Colocalization strategy unveils an underside binding site in the transmembrane domain of smoothened receptor. J Med Chem DOI: 10.1021/acs.jmedchem.9b00283 [published ahead of print].

Address correspondence to: Dr. Gunnar Schulte, Karolinska Institutet, Department Physiology and Pharmacology, Section Receptor Biology and Signaling, Solnavägen 9, S-171 65 Stockholm. E-mail: gunnar.schulte@ki.se 